\title{
Intelligent Learning First Aid (ILFA BOT)
}

\author{
Mazlina Md Mustaffa \\ mazlina_mustafa@puo.edu.my \\ Lecturer, Department of Information and Communication Technology, Polytechnic Ungku Omar, Malaysia
}

\begin{abstract}
This project focused on Artificial Intelligent (AI) and a chatbot represents machine that answers questions using Natural Language Processing (NLP) and allows the user to start a conversation with the machine. This paper presents the intelligent learning system for first aid called ILFA bot that aims to create intelligent virtual human agents to serve the role of online first aid guide for students. The research only covers the topic of First aid and the modules in ILFA website are Home, About Us, Contact and Video. The topics covers in First Aid are burns and scalds, bleedings, bone fractures, poisoning and Cardio Pulmonary Resuscitation (CPR). This web is a responsive web. Responsive web design (RWD) is a web development approaches that creating a dynamic changes to the appearance of a website, depending on the screen size and orientation of the device being used to view it. The different this web to other learning website is student can communicate with our bot namely ILFA to ask about First Aid. The students will be able to learn about the First Aid in an interactive way with all the information video and if there are any further inquiries, they can communicate with the ILFA Bot.The architecture that use in this research is 3-tiers technology and a waterfall model as a methodology in this project. To test the function of ILFA bot, the researcher uses Black Box testing. The testing is based on chatterbox challenge and questions. The result from testing shows that ILFA Bot get 358 out of 500 points, $24 \%$ from the points is full mark from overall question.
\end{abstract}

"Keywords: First Aid; chatterbot; artificial intelligent; renponsive web design."

\section{Introduction}

This paper presents the intelligent learning system for first aid called ILFA bot that aims to create intelligent virtual human agents to serve the role of online first aid guide for students. This research is focused on the polytechnic students who take AW101 and the researchers highlight for one topic only which is First Aid. The current method in teaching and learning process ( $\mathrm{T} \& \mathrm{~L}$ ) is not interactive and the students cannot deal affectively to study the topic. First aid is one of the topics in AW101. This course is compulsory for all students in polytechnic. First aid is very important topic that students should learn and know to identify first aid and safe work practice in order to maintain healthy and safe work environment. The students can practice the skills to respond for an accident action. Communicative tools are an important means by which VLEs have the potential to transform learning with computers from being passive and trans missive in nature (Sujana Jyothi, Claire McAvinia, 2012). 
From the observation, the polytechnic students have difficult to find the information about first aid in the context of learning for the course Aw101. The existing learning for the first aid technique does not interactive and the students cannot deal affectively. The current method that lecturers use is power point notes and these notes were uploaded in Curriculum Information Document Online System (CIDOS). CIDOS is Polytechnic ELearning Portal. The users have to register before using the CIDOS. It does not user friendly since they have to $\log$ in each time to use. The performance of existing techniques is not evaluated thoroughly. We educate our students in new ways, and we are seeing paradigm shifts in government, healthcare, and entertainment (Lester \& Mott, 2004).

A Chatbot is a program which allows the user to start a conversation with the machine. This is a platform focused on Artificial Intelligence (AI), which can be developed as messaging applications, web applications, or smartphone applications. A chatbot represents machine that answers questions using Natural Language Processing (NLP) (Chetan Bulla, 2010).

\section{Literature Review}

In this part, it focuses on the study, review and examines the related work about the chatterbot system are some researches which are relevant to domain specific knowledge the chatterbot system. There are a number of research works on the chatterbot.

For this research, domain specific knowledge system is a set knowledge base consisting of Artificial Intelligence Markup Language (AIML) files. Artificial Linguistic Internet Computer Entity (A.L.I.C.E) is an award winning open source natural language artificial intelligence chat robot which utilizes AIML to form responses to queries. The main knowledge bases of ALICE are stored on different AIML files; ALICE is available to the public for free under the GNU license (Johan Rahman, 2007).

Johan Rahman (Johan Rahman, 2007) implemented ALICE chat bot as domain specific knowledge for BRAU U (FAQ bot). This chat bot is a undergraduate student information system that helps students in BRAC University with admission and course information's. In particular, the proposal investigates the implementation of ALICE chat bot system as a domain specific chatterbot named FAQ bot, our work will show how a chat bot can work as domain specific information system and experiments on how the system's accuracy could be improved based on a specific domain.

The researchers, (Aw \& Lee, 2012) developed personalized chat normalizer for English and integrate it with a multilingual chat system allowing user to create and use personalized short forms in multilingual chat.

James Smith (Smith, 2010) created a chatterbot based interactive question-answering system capable of pronominal anaphora in a user-driven dialogue. The intention is that a user will be able to collect data on a given subject faster and more reliably than they would use a regular search engine and/or an existing QA service.

Ong Sing Goh (Ong Sing Goh, 2008) developed a conversation agent or conversation robot. Conversation agents are software programs which can be used to provide a natural interface between human and computers. Basically, it is a smart and effective communication technology where autonomous agent is able to carry out simulated human conversation via multiple channels. 


\section{Methodology}

This project use waterfall model as our methodology as shown in Figure 1.

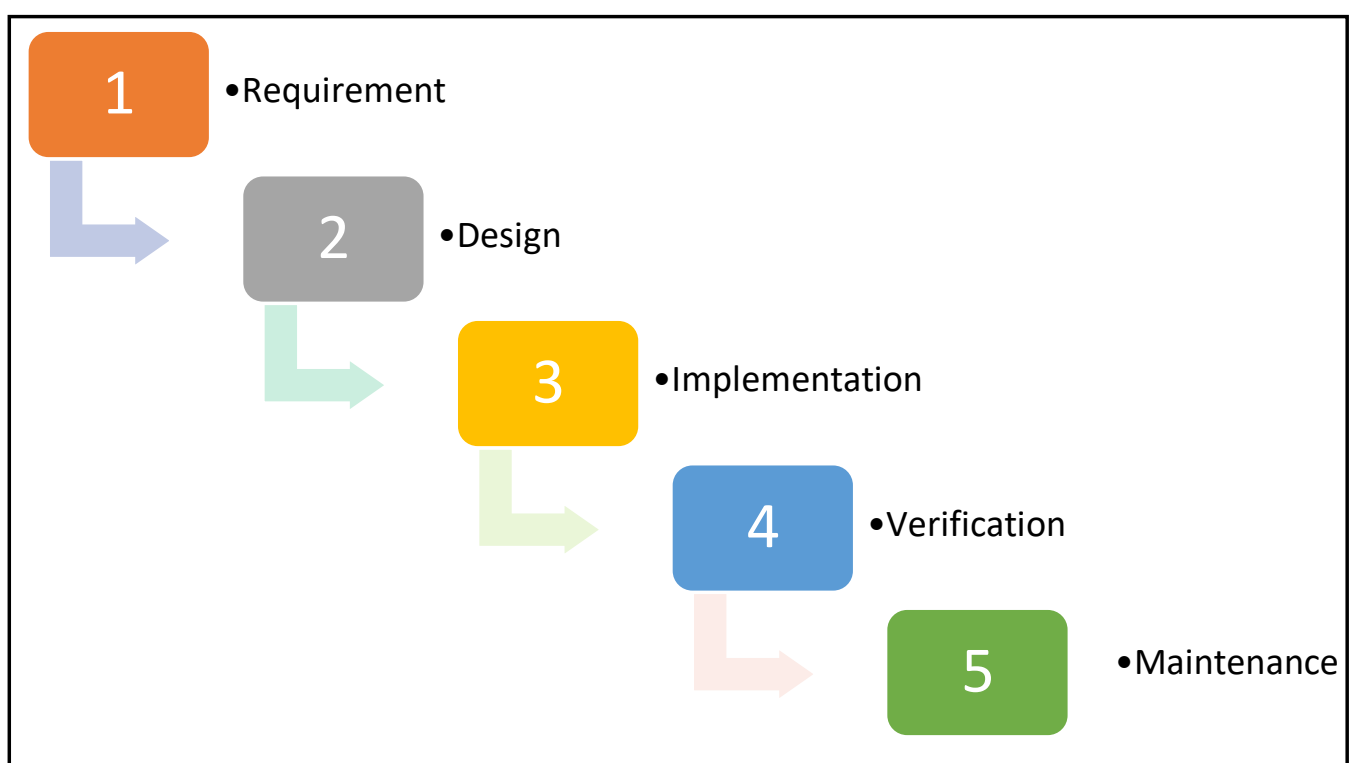

Fig. 1. : Waterfall Model

\subsection{Requirement}

During this stage, the business problem starts to be defined in greater detail. The first and most obvious element of the analysis stage is the collection of business requirements. Through a combination of techniques such as one-on-one discussions, group sessions, questionnaires, and surveys, the project team determines what the business needs are.

\subsection{Design}

The design phase is where the business requirements start to be translated into an IT solution.

- Design the storyboard of ILFA website's structure. It maps out all the components of the site and how it's interrelated. It is create to plan and organize the website and also plan the internal linking structure between pages.

- Design the data modelling of website and chatterbot. The design include Conceptual Design, Logical Design and Physical Design 


\subsection{Implementation}

In this phase the source code of the programs is written. The web is going to develop and to integrate with the bot which is called ILFA Bot. The Website creates using PHP and the Chatter Bot in Program O.

\subsection{Verification}

At this phase, the whole design and its construction is put under a test to check its functionality. If there are any errors then they will surface at this point of the process. Black-box testing is a method of software testing that examines the functionality of an application without peering into its internal structures or workings.

\subsection{Maintenance}

Maintenance and management is needed to ensure that the system will continue to perform as desired.

\section{Design}

The architecture that use in this research is 3-tiers technology. Then, it continues with the modules of function, storyboard for the web, navigation and data modelling. Finally, the last section will describe about the data flow diagram (DFD).

\subsection{System Architecture}

The technology of Artificial Intelligence Markup Language (AIML) has the ability to develop natural language agents or bots. AIML seems very fit for purpose as it was originally designed for conversational chatterbot. Numerous chatbot have been designed with the use of AIML as a dialogue manager including the original AIML chatbot ALICE (Smith, 2010).

The Fuzzy set Logic (FSL) can be affected and adopted for human and robot interaction problems since the human knowledge can be expressed by fuzzy sets and rules. It can make a machine reason in a simple that human reason about uncertain and ambiguous. The framework of ILFA bot is built with the application domain specific knowledge and natural language query.

Besides that, ILFA bot engine can response naturally and communicate in numerous independent conversation. ILFA bot uses the 3-tiers architecture technology. Three-tier architecture is a client-server architecture in which the functional process logic, data access, computer data storage and user interface are developed and maintained as independent modules on separate platforms. Three-tier architecture is a software design pattern and well-established software architecture. The three tiers are in presentation tier, application tier and data tier. The architecture of this system illustrated in Figure 2. 


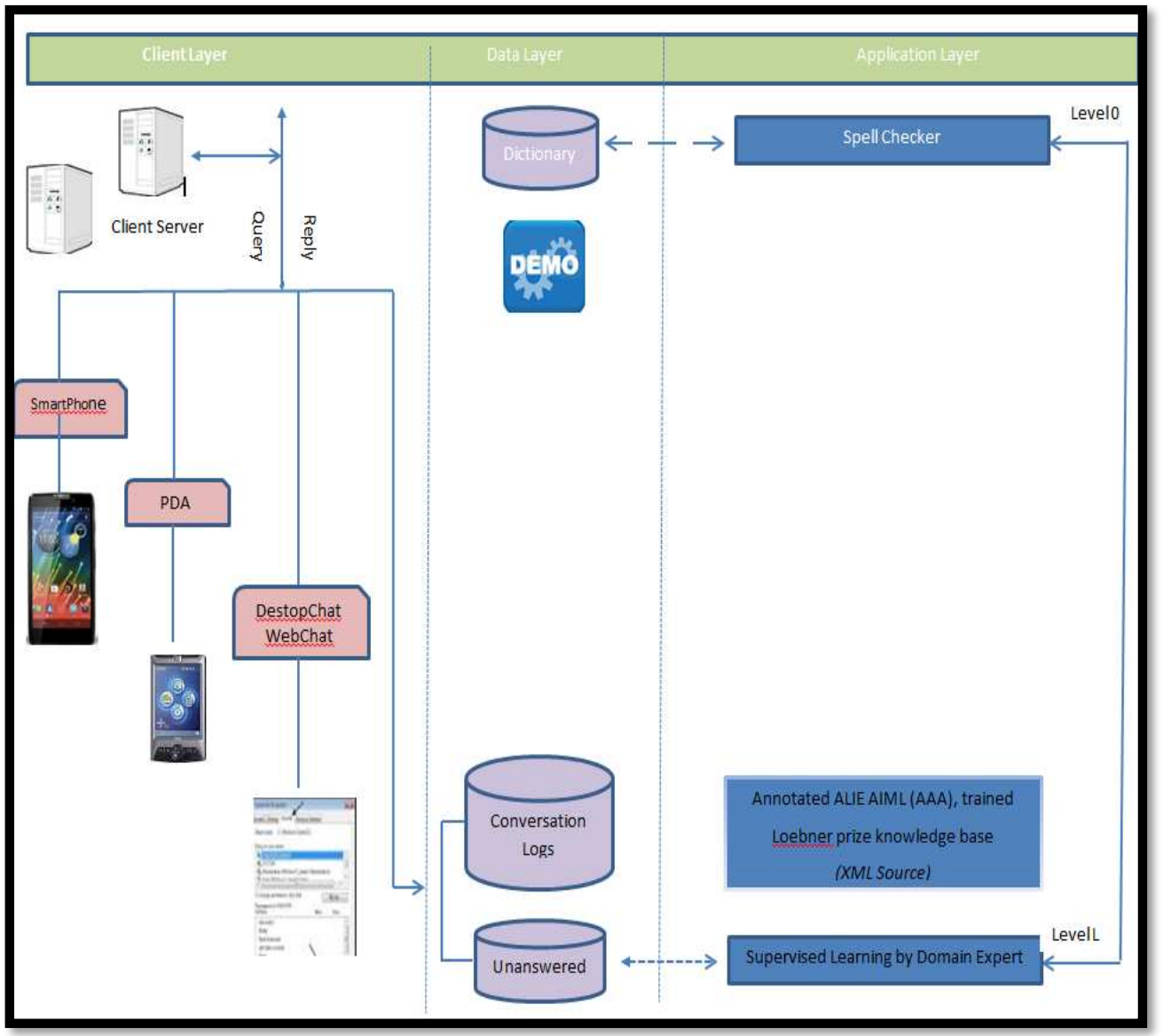

Fig. 2.: Architecture

- AIML based on client makes a request to a server

- Server will connect with the Knowledge base/AIML Brain File

- Server gets the appropriate response from the AIML Brain File

- Server forwards the response to the client

\subsection{Data Modelling}

In the design phase, the construction of this website and chatterbot has been shown in Conceptual Design, Logical Design and Physical Design.

- Conceptual Model Design - Conceptual Design will show in Figure 3. It shows the illustration how the data is structured. 


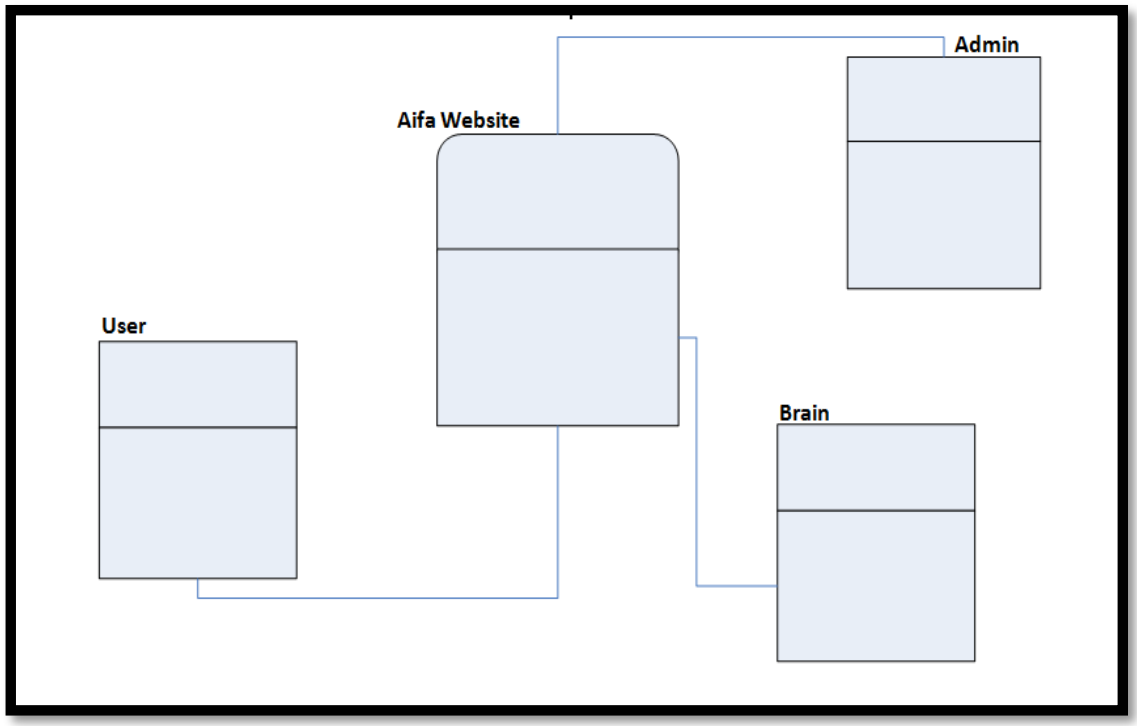

Fig. 3. : Data Modelling: Conceptual Model Design

- Logical Model Design - In Logical Design, it shows the table and the field for each data as shown in Figure 4.

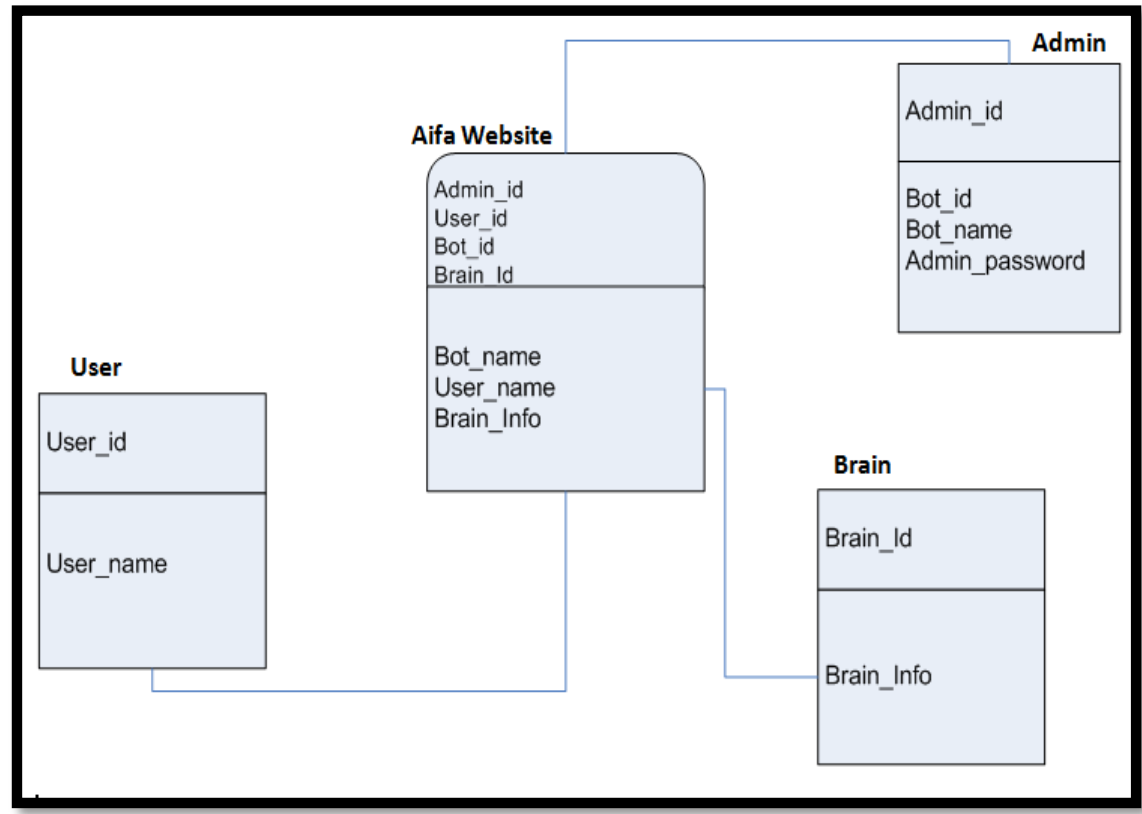

Fig. 4. : Data Modelling: Logical Model Design 
- $\quad$ Physical Model Design - Detail in the data type for each field id clearly illustrated in Figure 5.

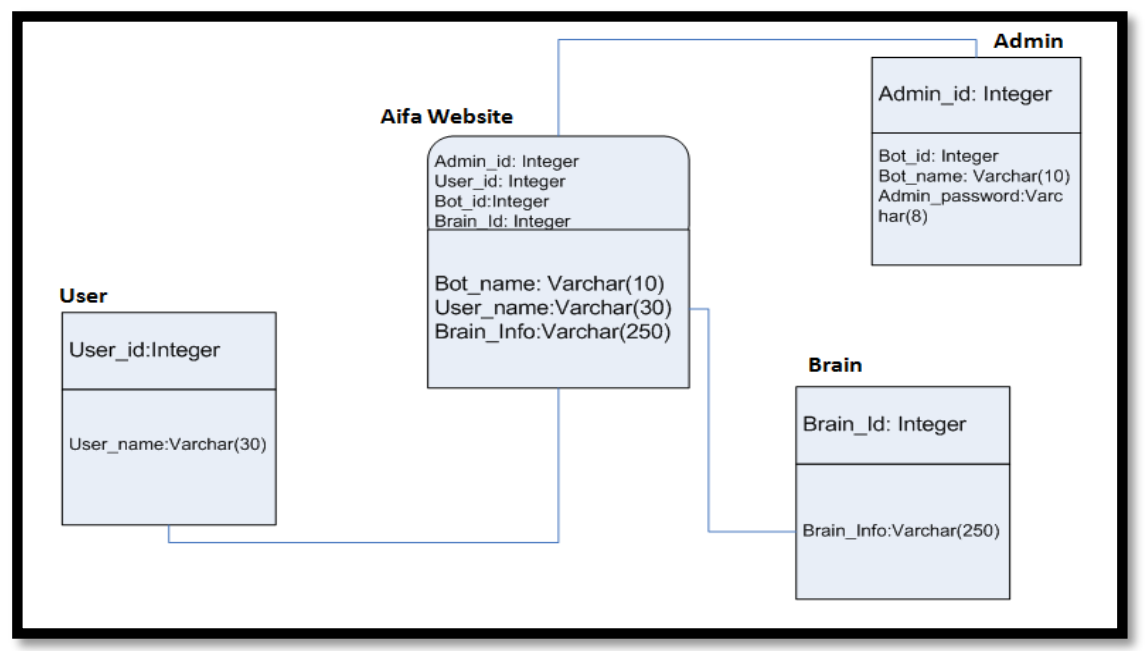

Fig. 5. : Data Modelling: Physical Model Design

\subsection{Data Flow Diagram (DFD)}

Dataflow diagram (DFD ) describing the system as a network of inter-related functions with one another. As shown in Figure 6, DFD context diagram on the application, which consists of three processes; login, giving question from user and process answer by ILFA bot.

- Admin log in and upload brain in Program O.

- User give question to ILFA bot

- $\quad$ ILFA bot process the answer and give feedback to user.

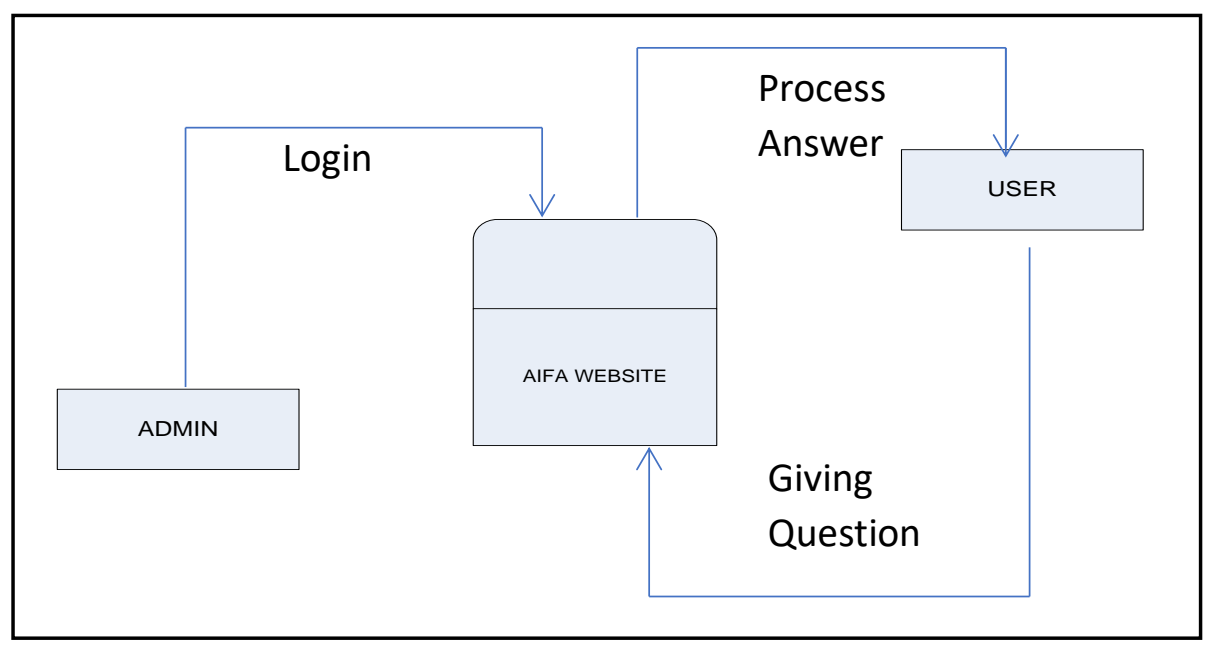

Fig. 6. : Data Flow Diagram 


\subsection{User Interface: Navigation}

Navigation diagrams of this website have been illustrated for better understanding of the web that are going to be developed and to integrated with the bot which is called AIFA Bot. It is shown in Figure 7.

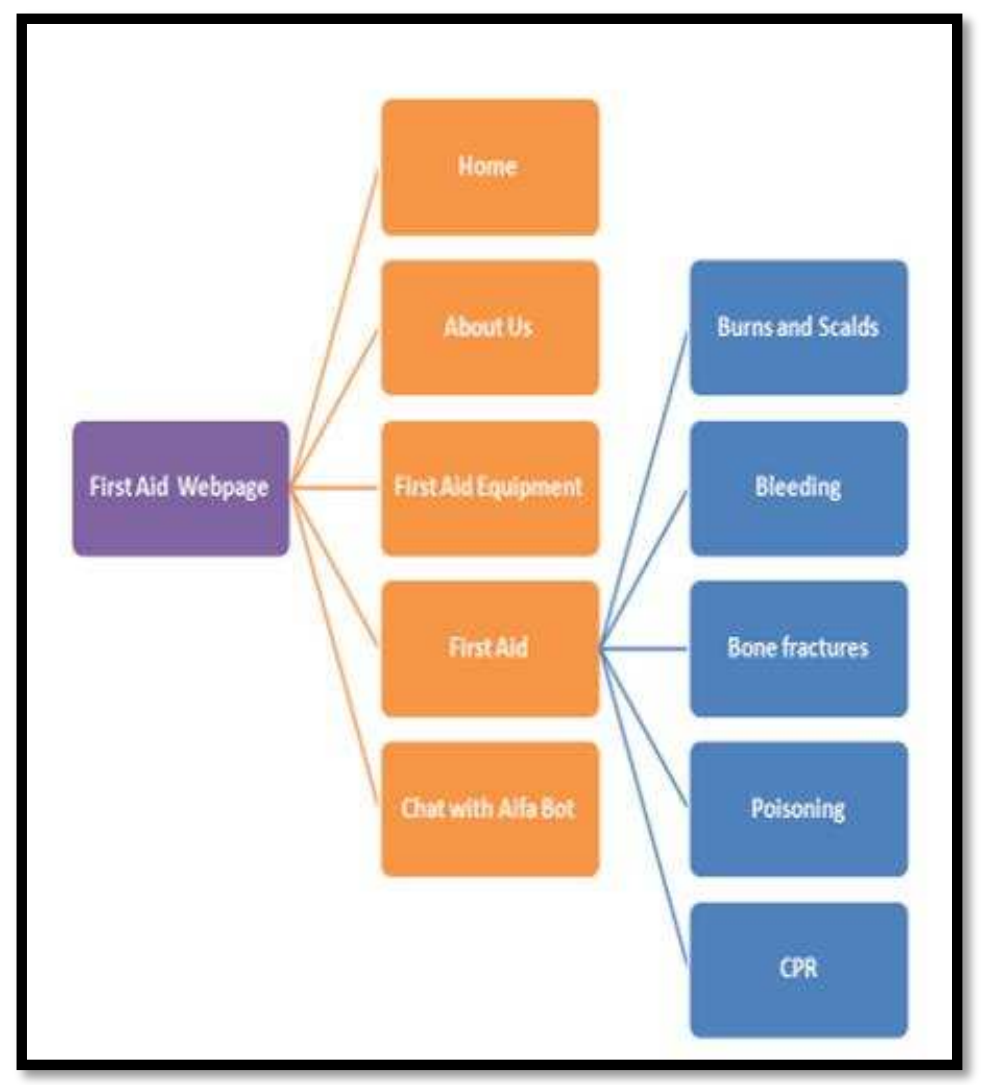

Fig. 7. : Navigation for ILFA web

\subsection{User Interface : Storyboard}

This storyboard is a visual representation of ILFA website's structure. It maps out all the components of the site and it's interrelating. It is create to plan and organize the website and also plan the internal linking structure between pages. It is shown in Figure 8 : Story Board. 


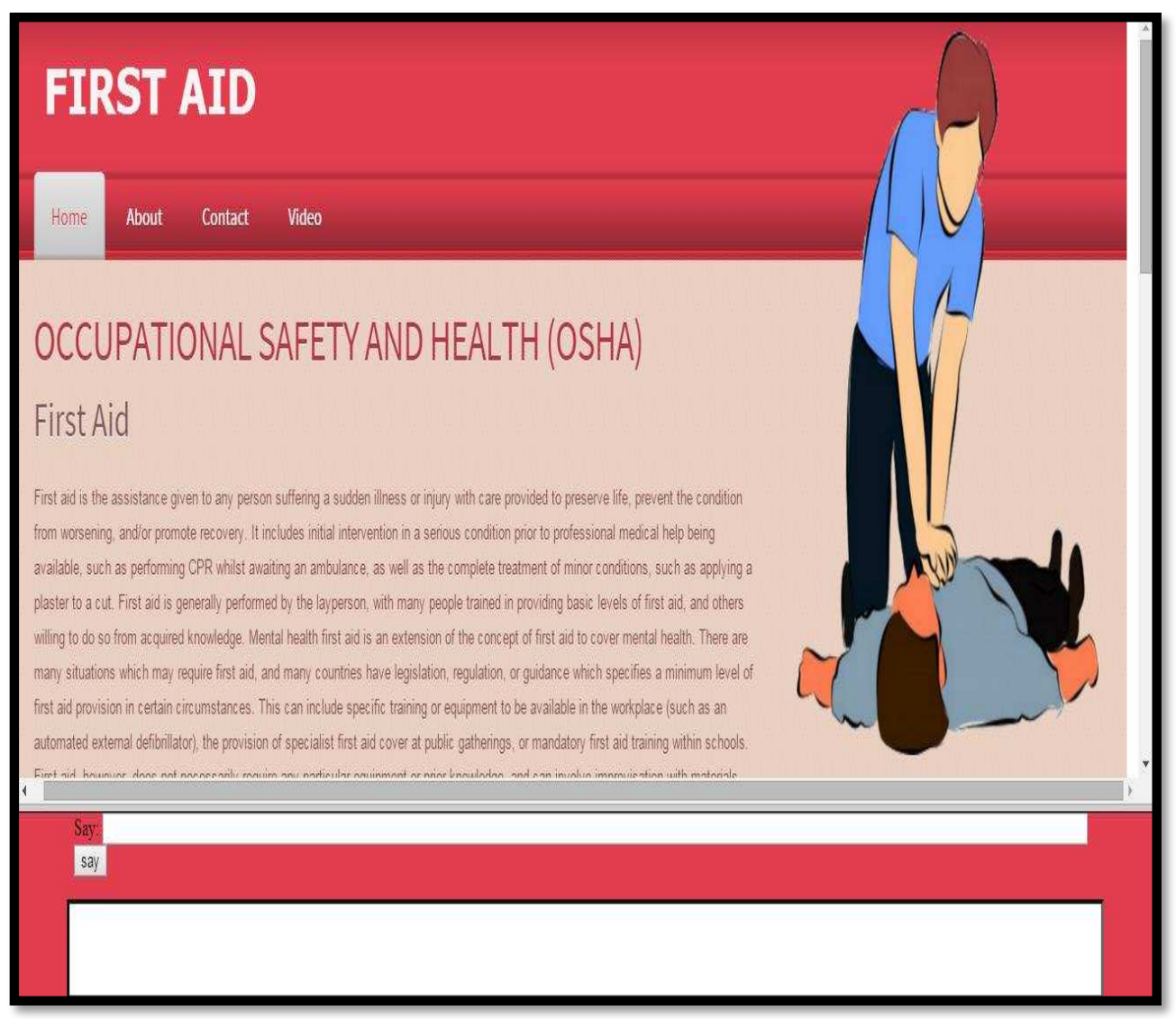

Fig. 8. :Story Board ILFA

\section{Analysis}

In this part, it will discuss about the analysis problem, experimental setup or design, the tools and technique we approach for this research, dataset we use and the measurement and validation. We use the black-box testing for the technique approach and the dataset we take from the related website.

\subsection{Dataset}

The dataset used to do the process of experiments and the chatlogs collected from the communications between users and chatbot retrieved based on particular topics such as CPR, basic first aid and basic first aid equipment of chat sessions. In this study, we also used the dataset from the previous competition www.chatterboxchallenge.comand http://loebner.net/Prizef/2001_Contest/Richard_Wallace.txt as shown in Table 1. 
Table 1:Questions from http://www.chatterboxchallenge.com/

\begin{tabular}{|c|l|}
\hline ITEM & \multicolumn{1}{|c|}{ QUESTIONS } \\
\hline 1. & What do you do when you get hungry? \\
\hline 2. & Is 4 an odd number? \\
\hline 3. & What is 67 divided by 26? \\
\hline 4. & What does bisexual mean? \\
\hline 5. & My mother has cancer. \\
\hline 6. & What kind of things does u like to do? \\
\hline 7. & Do you like convicted murderers? \\
\hline 8. & Who is the creator of the universe? \\
\hline 9. & What is the most expensive thing you bought? \\
\hline 10. & Tell me something interesting. \\
\hline 11. & Is sugar sweet or sour? \\
\hline 12. & When was the last time you saw a doctor? \\
\hline 13. & What's the main ingredient in apple pie? \\
\hline 14. & Where is Tokyo located? \\
\hline 15. & I think you're very smart. \\
\hline
\end{tabular}

\subsection{Measurement / Validation}

In this research, chatterbot system will be measured for its consistency. The accuracy of this system will be measured on chatterbot responds to the questions from the users which is knowledge domain. The chatterbot also are measured for its stability which is based on the correct answers in 24/7 hours. Basically, it is measured based on the answers from the similar topic questions will be different but still in the topic range. The chatterbot will give the same answers according to those questions.

\section{Evaluation}

Software quality is an important part of software development. Software quality assurance (SQA) is a measurement mechanism that is applied throughout the software engineering process. Software testing is the most important phase in software quality assurance. The major effort in software engineering is spent after development on maintaining systems in order to remove existing errors and to adapt the systems to changed requirements. Software testers often spend considerable energy trying to recover the design information before testing a program. Without an adequate understanding of a program's structure, it is difficult to maintain it effectively.

Software testing is a critical component of the software engineering process. It is an element of software quality assurance and can be described as a process of running a program in such a manner as to uncover any errors. At this phase, the whole design and its construction is put under a test to check its functionality. If there are any errors then they will surface at this point of the process 


\subsection{Method for Software Testing}

There are different methods which can be used for Software testing:

\section{- $\quad$ Black Box Testing}

The technique of testing without having any knowledge of the interior workings of the application is Black Box testing. The tester is oblivious to the system architecture and does not have access to the source code. Typically, when performing a black box test, a tester will interact with the system's user interface by providing inputs and examining outputs without knowing how and where the inputs are worked upon. The main purpose of BB Testing is to validate that the application works as the user will be operating it and in the environments of their systems. Black-box testing checks that the user interface and user inputs and outputs all work correctly. Part of this is that error handling must work correctly. It's used in functional and system testing.

\section{- White Box Testing}

White box testing is the detailed investigation of internal logic and structure of the code. White box testing is also called glass testing or open box testing. In order to perform white box testing on an application, the tester needs to possess knowledge of the internal working of the code. The tester needs to have a look inside the source code and find out which unit/chunk of the code is behaving inappropriately.

\section{- $\quad$ Grey Box Testing}

Grey Box testing is a technique to test the application with limited knowledge of the internal workings of an application. In software testing, the term the more you know the better carries a lot of weight when testing an application. Mastering the domain of a system always gives the tester an edge over someone with limited domain knowledge. Unlike black box testing, where the tester only tests the application's user interface, in grey box testing, the tester has access to design documents and the database. Having this knowledge, the tester is able to better prepare test data and test scenarios when making the test plan. In this study, we used black box testing which is black-box testing checks that the user interface and user inputs and outputs all work correctly. Part of this is that error handling must work correctly. It's used in functional and system testing.

\subsection{Interface Mobile}

Responsive web design (RWD) is an approach to web design aimed at crafting sites to provide an optimal viewing experience easy reading and navigation with a minimum of resizing, panning, and scrolling across a wide range of devices (from desktop computer monitors to mobile phones). This project use Browser Stack to test website on any screen size including desktops, tablets, televisions, and mobile phones. 


\subsection{Analysis Of Chat}

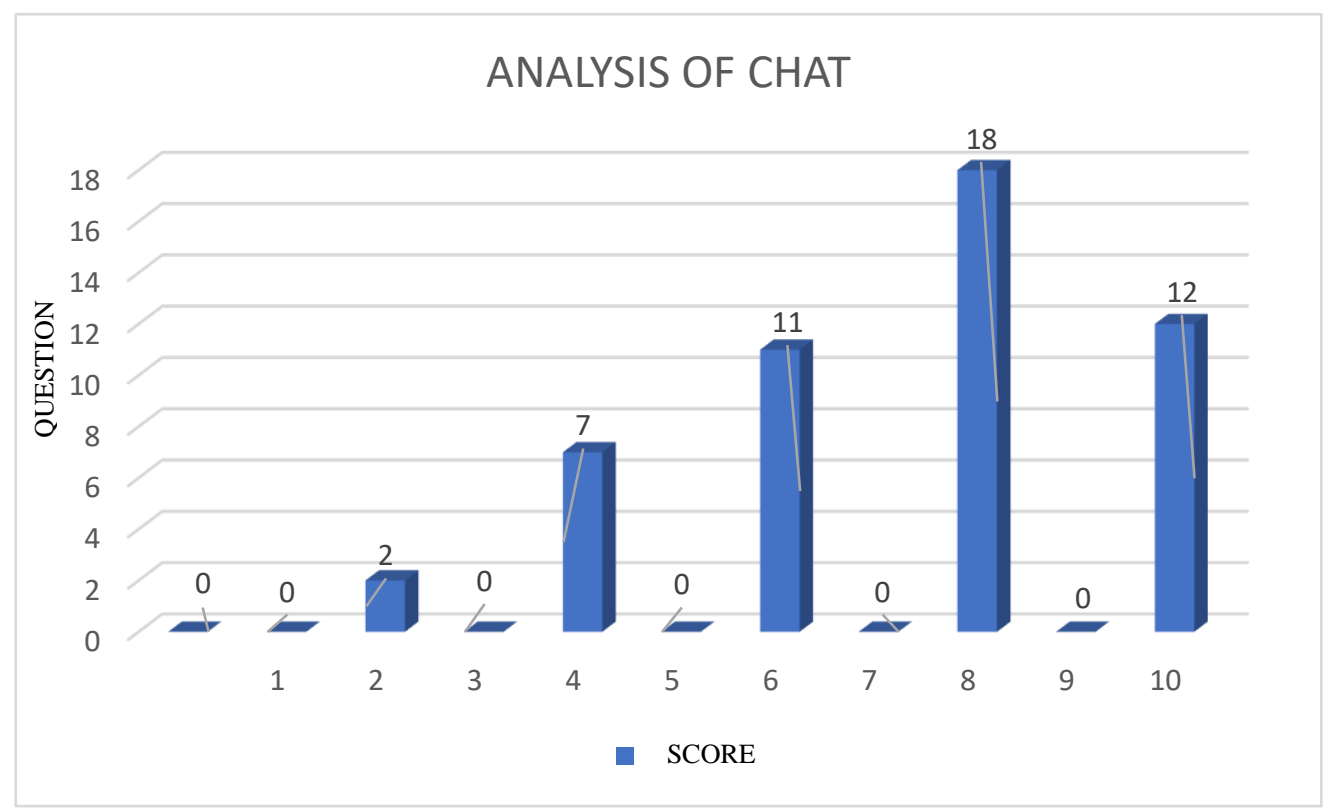

Fig. 7. : Analysis Of Chat

Figure 7 show the analysis made on the ILFA bot. After testing, we found this bot got 358 marks out of 500marks. Of the 50 questions that were asked 12 questions bot got 10 points, 18 questions got 8 points, 11questions scored 6 points, 7 questions have 4 points, and 2 questions got 2 points.

\section{Conclusion}

Techniques from artificial intelligence, computer animation, and human-computer interaction are increasingly converging in the field of embodied conversational agents. Conversational agents have been produced to meet a wide range of applications, including tutoring, question-answering, conversation practice for language learners, pedagogical agents and learning companions, and dialogues to promote reflection and meta-cognitive skills.

The research only covers the topic of First aid in AW101. The modules in ILFA website are Home, About Us, Contact and Video. The topics covers in First Aid are burns and scalds, bleedings, bone fractures, poisoning and Cardio Pulmonary Resuscitation (CPR). This web is a responsive web. Responsive web design (RWD) is a web development approaches that creating a dynamic changes to the appearance of a website, depending on the screen size and orientation of the device being used to view it. The different this web to other learning website is student can communicate with our bot namely ILFA to ask about First Aid. The students will be able to learn about the First Aid in an interactive way with all the information video and if there are any further inquiries, they can communicate with the ILFA Bot. To test the function of ILFA bot, the researcher uses Black Box testing. The testing is based on chatterbox challenge and questions. The result from testing shows that ILFA Bot get 358 out of 500 points, $24 \%$ from the points is full mark from overall question. 


\section{Acknowledgements}

We would like to thank ICT Department, PUO for allowing me to conduct this study and my greatest appreciation to all participants who had participated in this study.

\section{References}

Chetan Bulla, Chinmay P., Akshata T., Samiksha A., Sachin K., 2010. A Review of AI Based Medical Assistant Chatbot, Research and Applications of Web Development and Design.

Sujana Jyothi, Claire McAvinia, J. K., 2012. A visualisation tool to aid exploration of students' interactions in asynchronous online communication. Computers \& Education Volume 58, Issue 1, January 2012, Pages 30-42.

Lester, J., \& Mott, B., 2004. Conversational Agents, 1-17.

Johan Rahman., 2007. Implementation of ALICE chatbot as domain specific knowledge bot for BRAU U (FAQ bot).

Aw, A. T., \& Lee, L. H., 2012. Personalized Normalization for a Multilingual Chat System, (July), 31-36.

Smith, J., 2010. IQABOT : A Chatbot-Based Interactive Question-Answering System, (April).

Ong Sing Goh, 2008. A framework and Evaluation of Conversation Agents. 